\title{
Emergence of Very Broad Infrared Absorption Band by Hyperdoping of Silicon with Chalcogens
}

\section{Citation}

Umezu, Ikurou, Jeffrey M. Warrender, Supakit Charnvanichborikarn, Atsushi Kohno, James S. Williams, Malek Tabbal, Dimitris G. Papazoglou, Xi-Cheng Zhang, and Michael J. Aziz. 2013. Emergence of Very Broad Infrared Absorption Band by Hyperdoping of Silicon with Chalcogens. Journal of Applied Physics 113(21): 213501.

\section{Published Version}

doi:10.1063/1.4804935

\section{Permanent link}

http://nrs.harvard.edu/urn-3:HUL.InstRepos:17190516

\section{Terms of Use}

This article was downloaded from Harvard University's DASH repository, and is made available under the terms and conditions applicable to Other Posted Material, as set forth at http:// nrs.harvard.edu/urn-3:HUL.InstRepos:dash.current.terms-of-use\#LAA

\section{Share Your Story}

The Harvard community has made this article openly available.

Please share how this access benefits you. Submit a story.

\section{Accessibility}




\title{
Emergence of very broad infrared absorption band by hyperdoping of silicon with chalcogens
}

\author{
Ikurou Umezu, ${ }^{1}$ Jeffrey M. Warrender, ${ }^{2}$ Supakit Charnvanichborikarn, ${ }^{3}$ Atsushi Kohno, ${ }^{4}$ \\ James S. Williams, ${ }^{3}$ Malek Tabbal, ${ }^{5}$ Dimitris G. Papazoglou, ${ }^{6,7}$ Xi-Cheng Zhang, ${ }^{8, a)}$ \\ and Michael J. Aziz ${ }^{9}$ \\ ${ }^{1}$ Department of Physics, Konan University, Kobe 658-8501, Japan \\ ${ }^{2}$ U.S. Army ARDEC-Benét Laboratories, Watervliet, New York 12189, USA \\ ${ }^{3}$ Research School of Physics and Engineering, The Australian National University, Canberra, ACT 0200, \\ Australia \\ ${ }^{4}$ Department of Applied Physics, Fukuoka University, Fukuoka 814-0180, Japan \\ ${ }^{5}$ Department of Physics, American University of Beirut, Beirut 1107 2020, Lebanon \\ ${ }^{6}$ Institute of Electronic Structure and Laser, Foundation for Research and Technology Hellas, P.O. Box 1527, \\ 71110 Heraklion, Greece \\ ${ }^{7}$ Materials Science and Technology Department, University of Crete, P.O. Box 2208, 71003 Heraklion, Greece \\ ${ }^{8}$ Department of Physics, Applied Physics and Astronomy, Rensselaer Polytechnic Institute, Troy, \\ New York 12180, USA \\ ${ }^{9}$ Harvard School of Engineering and Applied Sciences, Cambridge, Massachusetts 02138, USA
}

(Received 9 September 2012; accepted 29 April 2013; published online 3 June 2013)

We report the near through mid-infrared (MIR) optical absorption spectra, over the range $0.05-1.3 \mathrm{eV}$, of monocrystalline silicon layers hyperdoped with chalcogen atoms synthesized by ion implantation followed by pulsed laser melting. A broad mid-infrared optical absorption band emerges, peaking near $0.5 \mathrm{eV}$ for sulfur and selenium and $0.3 \mathrm{eV}$ for tellurium hyperdoped samples. Its strength and width increase with impurity concentration. Its strength decreases markedly with subsequent thermal annealing. The emergence of a broad MIR absorption band is consistent with the formation of an impurity band from isolated deep donor levels as the concentration of chalcogen atoms in metastable local configurations increases. (C) 2013 AIP Publishing LLC. [http://dx.doi.org/10.1063/1.4804935]

\section{INTRODUCTION}

Silicon hyperdoped with chalcogens can be synthesized by pulsed laser irradiation in a sulfur-bearing atmosphere, ${ }^{1,2}$ ion implantation followed by pulsed laser melting, ${ }^{3,4}$ or pulsed laser mixing. ${ }^{5}$ This material has attracted interest because of its sub band gap absorption and has been studied as a candidate for infrared (IR) photodetectors ${ }^{6-8}$ and efficient solar cells. ${ }^{9-11}$ In addition, observations of carrier lifetime recovery for sufficiently high concentrations of titanium in silicon has aroused similar interest in this material. ${ }^{12,13}$

Chalcogen hyperdoping has been shown to cause an insulator-to-metal transition and has been proposed to form an intermediate band in the silicon band gap. ${ }^{4,10,14-16}$ However, the hyperdoping-induced changes causing the sub-bandgap absorption have remained unclear. One reason for this is that most measurements of optical absorption have been limited to the near IR (NIR) region, or to photon energies above the band gap, ${ }^{17}$ even though the IR absorption band is extremely broad. In this paper, we report measurements of the optical absorption coefficient over a broad range of photon energies spanning near- to mid-IR (MIR) wavelengths. The results are consistent with the formation of an impurity band in the silicon band gap. We also report the effects of impurity dose and of post-processing thermal treatment in selected cases.

\footnotetext{
a)Present address: The Institute of Optics, University of Rochester, Rochester, New York 14627, USA.
}

\section{EXPERIMENT}

Double side polished p type (001) Si wafers, resistivity of 5-25 $\Omega \mathrm{cm}$, were ion implanted at room temperature with either $95 \mathrm{keV}{ }^{32} \mathrm{~S}^{-}, 176 \mathrm{keV}{ }^{80} \mathrm{Se}^{+}$, or $245 \mathrm{keV}{ }^{130} \mathrm{Te}^{+}$to doses of $1 \times 10^{16}$ ions $/ \mathrm{cm}^{2}$. The dose of ${ }^{32} \mathrm{~S}^{-}$was varied from $3 \times 10^{14}$ to $1 \times 10^{16}$ ions $/ \mathrm{cm}^{2}$ and pre-amorphized by $85 \mathrm{keV} \mathrm{Si}^{-}$to doses of $3 \times 10^{15}$ ions $/ \mathrm{cm}^{2}$ when the ${ }^{32} \mathrm{~S}^{-}$ dose is not greater than $1 \times 10^{15}$ ions $/ \mathrm{cm}^{2}$. Pulsed laser melting was performed using a $\mathrm{XeCl}$ excimer laser beam (308 nm, $25 \mathrm{~ns}$ FWHM, $50 \mathrm{~ns}$ total duration). Each sample received three laser shots at $1.7 \mathrm{~J} / \mathrm{cm}^{2}$ followed by a fourth laser shot at $1.8 \mathrm{~J} / \mathrm{cm}^{2}$. Time-resolved reflectivity of a $488 \mathrm{~nm} \mathrm{Ar}^{+}$ion laser was used to measure the melt duration. The laser fluence was calibrated by comparing the melt duration with numerical solutions to the one-dimensional heat equation. ${ }^{18}$ The details of the sample preparation method and depth profiles of chalcogen atoms observed by secondary ion mass spectrometry (SIMS) are reported elsewhere. ${ }^{3}$ For all samples in this paper, the synthesis procedure is the same as that in the previous work for sulfur doped samples, whereas the previous work employed a somewhat higher fluence for Se and Te doped samples. Following laser melting, some of the samples were thermally annealed in a flowing argon gas atmosphere for $30 \mathrm{~min}$.

We measured the transmittance and reflectance of samples in the NIR region using a grating spectrophotometer equipped with an integrating sphere detector. The measured 
reflectance, $R$, of the hyperdoped samples in the NIR region was similar to that calculated by using published optical constants of pure $\mathrm{Si}^{19}$ when taking into account multiple reflection at the front and back surfaces. This result suggests that under conditions described in the following paragraph, where we cannot obtain experimental values of $R$ for hyperdoped materials, the error may be acceptably small if we compute it using the reflectivity at a single surface, $r$, of pure Si calculated from published optical constants. The transmittance $T$ is described as follows, by taking into account multiple reflections at the front and back surfaces ${ }^{20}$

$$
T=\frac{(1-r)^{2} \exp (-\alpha d)}{1-r^{2} \exp (-2 \alpha d)},
$$

where $\alpha, d$, and $r$ are the absorption coefficient, the effective thickness of the absorbing layer, and reflectivity of a single surface, respectively. The $\alpha d$ product of this sample thus can be obtained as follows by using measured transmittance $T$ and reflectance $r$ of pure $\mathrm{Si}$

$$
\alpha d=-\ln \left(-\frac{(1-\mathrm{r})^{2}-\sqrt{(1-\mathrm{r})^{4}+4 \mathrm{r}^{2} \mathrm{~T}^{2}}}{2 r^{2} T}\right) .
$$

In this paper, we present our absorption results as $\alpha d$ rather than $\alpha$, because the impurity concentration in the hyperdoped layer is not constant as a function of depth, and $\alpha$ depends on the impurity concentration, as shown in Ref. 3. When $\alpha$ is necessary for analysis, we estimate the average $\alpha d$ of the hyperdoped layer by assuming the hyperdoped layer to be $250 \mathrm{~nm}$ thick, based on SIMS measurements. ${ }^{3}$ Similarly, dopant and carrier concentrations are estimated using this nominal thickness. Consequently, the peak concentration as a function of depth is somewhat higher than the reported average value.

A Fourier transform infrared (FTIR) spectrometer was used to measure MIR optical transmittance. We obtained $\alpha d$ in the MIR region for hyperdoped materials by using Eq. (2) and by assuming the reflectivity $r$ is equal to that for pure $\mathrm{Si}$ in the MIR region, as calculated using published optical constants of pure Si.

The carrier concentration was measured by spreading resistance profiling (SRP) for some samples. The conditions of ion implantation and pulsed laser melting are the same with that of the samples for optical measurement except for laser fluence. Samples containing sulfur, selenium and tellurium received four laser shots at $1.7,2.3$, and $2.3 \mathrm{~J} / \mathrm{cm}^{2}$, respectively. The measured resistivity $\rho$ was converted to carrier concentration $n$ using $n=1 / e \rho \mu$ with $e$ the electron charge and $\mu$ the electron mobility. The model of Kaiblinger-Grujin $e t$ al. ${ }^{21}$ was used to calculate the sulfur concentration-dependent mobility using the SIMS data to provide the atomic profiles.

The conditions of ion implantation and pulsed laser melting are listed in Tables I and II.

\section{RESULTS}

The effect of implanted sulfur dose on the optical absorption spectrum is shown in Fig. 1(a). The optical
TABLE I. Ion implantation conditions.

\begin{tabular}{lcc}
\hline \hline Ion & Energy $(\mathrm{keV})$ & Dose $\left(\mathrm{ions} / \mathrm{cm}^{2}\right)$ \\
\hline${ }^{32} \mathrm{~S}^{-}$ & 95 & $1 \times 10^{16}, 3 \times 10^{15}, 1 \times 10^{15}, 3 \times 10^{14}$ \\
${ }^{80} \mathrm{Se}^{+}$ & 176 & $1 \times 10^{16}$ \\
${ }^{130} \mathrm{Te}^{+}$ & 245 & $1 \times 10^{16}$ \\
\hline \hline
\end{tabular}

absorption spectrum of an as-implanted sample is not shown because the absorption was much smaller than that of pulsed laser melted samples. ${ }^{4}$ The discontinuity observed around $0.5 \mathrm{eV}$ is due to the change of spectrometer. The emergence and evolution of sub-bandgap absorption with implantation dose is clearly observed. These spectra include absorption of the substrate, below the implanted and laser melted region. For comparison, the measured ad spectrum of an unimplanted substrate is shown in the inset of Fig. 1(a). The signals between 0.15 and $0.25 \mathrm{eV}$ and between 0.36 and $0.5 \mathrm{eV}$ are not reliable for $\alpha d$ less than 0.01 due to absorption by air in the optical path. The increase in absorption above $1.0 \mathrm{eV}$ is due to the fundamental band-to-band absorption of the $\mathrm{Si}$ substrate, and absorption below $0.2 \mathrm{eV}$ is partially due to the free carrier absorption of the substrate. The contribution of free carrier absorption of the hyperdoped layer is discussed later. Because the optical absorption of the unimplanted substrate between 0.2 and $1.0 \mathrm{eV}$ is much smaller than that of the sulfur-doped samples, we may conclude that the broad absorption band which peaks at around $0.4-0.5 \mathrm{eV}$ is due to the presence of sulfur. Hereafter, we call this absorption band the MIR band. Although the absorption of the lowestdose sample is comparable to that of the unimplanted substrate below $0.6 \mathrm{eV}$, its broad absorption between 0.6 and $1.0 \mathrm{eV}$ is clearly distinguishable. Both the intensity and the width of the MIR band increase with the dose of initially implanted sulfur. We define the strength of the MIR band as the integral of the $\alpha d$ spectrum from 0.2 to $1.0 \mathrm{eV}$; it is
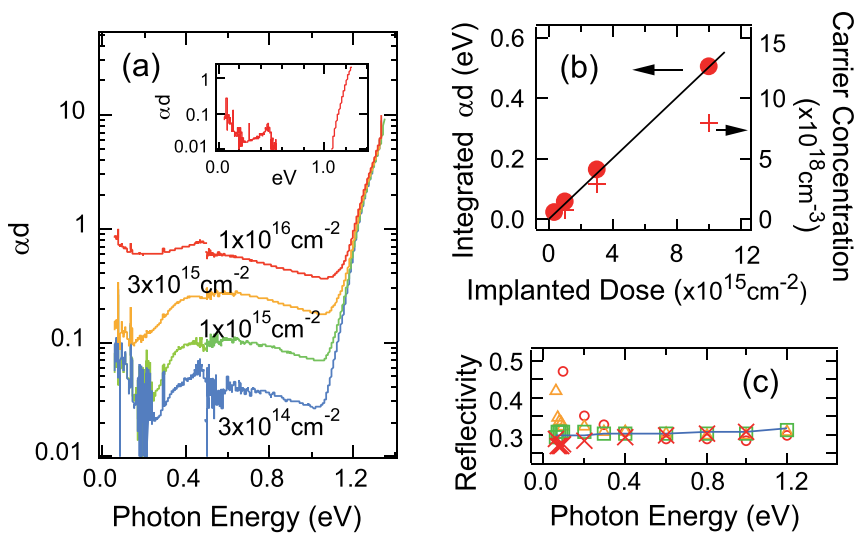

FIG. 1. (a) Effect of initially implanted sulfur dose on optical absorption spectra of laser hyperdoped samples. Implanted sulfur doses are indicated. The inset shows a measurement of an unimplanted substrate. (b) Integrated $\alpha d$ from 0.2 to $1.0 \mathrm{eV}$ (circles) and carrier concentration measured by Hall effect in Ref. 9 (crosses) as functions of implanted sulfur dose. (c) Calculated reflectivity by Kramers-Kronig transformation of the absorption spectrum. The blue line represents the literature value, and symbols correspond to $\mathrm{S}$ doses of $1 \times 10^{16}$ (red circles), $3 \times 10^{15}$ (orange triangles), and $1 \times 10^{15} \mathrm{~cm}^{-2}$ (green squares). Red crosses represent a $1 \times 10^{16} \mathrm{~cm}^{-2}$ dose sample annealed at $550^{\circ} \mathrm{C}$. 
shown as a function of initially implanted sulfur dose in Fig. 1(b) as solid circles. A linear correlation is observed between the strength of the MIR band and the initially implanted sulfur dose. The contribution to the MIR band of the $\mathrm{S}$ atoms based on the oscillator strength of the atoms is discussed in Sec. IV B.

The effect of conventional thermal annealing on the optical absorption of sulfur, selenium, and tellurium hyperdoped samples is shown in Figs. 2(a)-2(c), respectively. The ion-implanted dose for these samples was $1 \times 10^{16} \mathrm{~cm}^{-2}$. The spectra of the samples before thermal annealing (not shown in Fig. 2) were similar to those of the samples annealed at $250{ }^{\circ} \mathrm{C}$. The intensity of the MIR absorption band decreases with increasing annealing temperature for all samples. Sulfur concentration-depth profiles measured by SIMS showed no appreciable change for samples annealed at or below $550{ }^{\circ} \mathrm{C}$. Although we did not measure Se or Te concentration depth profiles, we do not expect different results from that of the sulfur-implanted sample due to an expected lower diffusivity. ${ }^{22}$

As discussed in the experimental section, in order to obtain $\alpha d$ we assumed that the reflectivity of the hyperdoped surface is the same as that of virgin $\mathrm{Si}$. When the optical absorption of the MIR band is small enough, this assumption is reasonable according to the Kramers-Kronig relationship between optical absorption and reflectivity. Because the optical absorption of the MIR band is not negligible in hyperdoped samples, it is necessary to question the validity of this assumption. It is reasonable at this stage to check for self-consistency between the assumed reflectivity and the resulting $\alpha d$ spectrum. The reflectivity was calculated by a Kramers-Kronig transformation of the absorption spectrum shown in Fig. 1(a) and the results are shown in Fig. 1(c). However, the $\alpha$ value depends on the depth due to the depth profile of dopant; we estimate the averaged $\alpha$ by assuming the thickness of the hyperdoped region as $250 \mathrm{~nm}$ according to the result of SIMS. ${ }^{3}$ The difference between reflectivity values thus calculated by the Kramers-Kronig method and the assumed reflectivity - that is, the reflectivity calculated using the optical constants of pristine $\mathrm{Si}-$ is at most a few percent for photon energies above $0.3 \mathrm{eV}$. This means that the method to obtain $\alpha d$ is self-consistent above $0.3 \mathrm{eV}$. The differences between the reflectivity calculated by the Kramers-Kronig method and the assumed reflectivity of pristine $\mathrm{Si}$ tend to increase with decreasing photon energy and they reach more than a few percent below $0.3 \mathrm{eV}$ and $0.1 \mathrm{eV}$ for the samples implanted with sulfur to $1 \times 10^{16} \mathrm{~cm}^{-2}$ and $3 \times 10^{15} \mathrm{~cm}^{-2}$, respectively. The differences are within a few percent for lower dose samples throughout the measured photon energy range. The deviations indicate that selfconsistency is not satisfied; hence the results for high dose samples are expected to be less reliable in these ranges. The large deviation of the $1 \times 10^{16} \mathrm{~cm}^{-2}$ dose sample is mainly caused by an increase in extinction coefficient, $\kappa$, at lower energy. Therefore, the deviation is smaller for the samples having lower $\alpha$ value. The samples that were annealed at $550^{\circ} \mathrm{C}$ and $700{ }^{\circ} \mathrm{C}$ show a much smaller deviation. We note that results of the Kramers-Kronig method are not perfect at lower photon energy, below about $0.1 \mathrm{eV}$, because we cannot measure optical absorption spectra below $0.05 \mathrm{eV}$.

\section{DISCUSSION}

\section{A. Optical absorption below $0.2 \mathrm{eV}$ and carrier concentration}

An increase in the value of $\alpha d$ with decreasing photon energy below $\sim 0.2 \mathrm{eV}$ is apparent in Figs. 1(a) and 2(a). Because the carrier concentration of the $\mathrm{S} 1 \times 10^{16} \mathrm{~cm}^{-2}$ hyperdoped sample is over $10^{19} \mathrm{~cm}^{-3}, 3,9$ it appears that free carrier absorption is responsible for the observed increase. We interpret the optical absorption data below $0.2 \mathrm{eV}$ as a superposition of the MIR band, contributing $(\alpha d)_{\mathrm{MIR}}$, and free carrier absorption, contributing $(\alpha d)_{\mathrm{fc}}$. To estimate the contribution of the tail of the MIR band in the overlapping region, we fitted a straight line to the $\alpha d$ spectra in Figs. 1(a) and 2 between 0.3 and $0.4 \mathrm{eV}$ and extrapolated it linearly down to $0.05 \mathrm{eV}$. The difference between the measured $\alpha d$ spectrum and the extrapolation is an estimate of the free carrier contribution $(\alpha d)_{\mathrm{fc}}$. To obtain $(\alpha d)_{\mathrm{fc}}, \alpha d$ of the underlying substrate, shown in the inset of Fig. 1(a), is subtracted from the spectra shown in Figs. 1(a) and 2. The subtracted spectra are not shown here because the effect of the substrate is very small for $1 \times 10^{16} \mathrm{~cm}^{-2}$ hyperdoped samples.
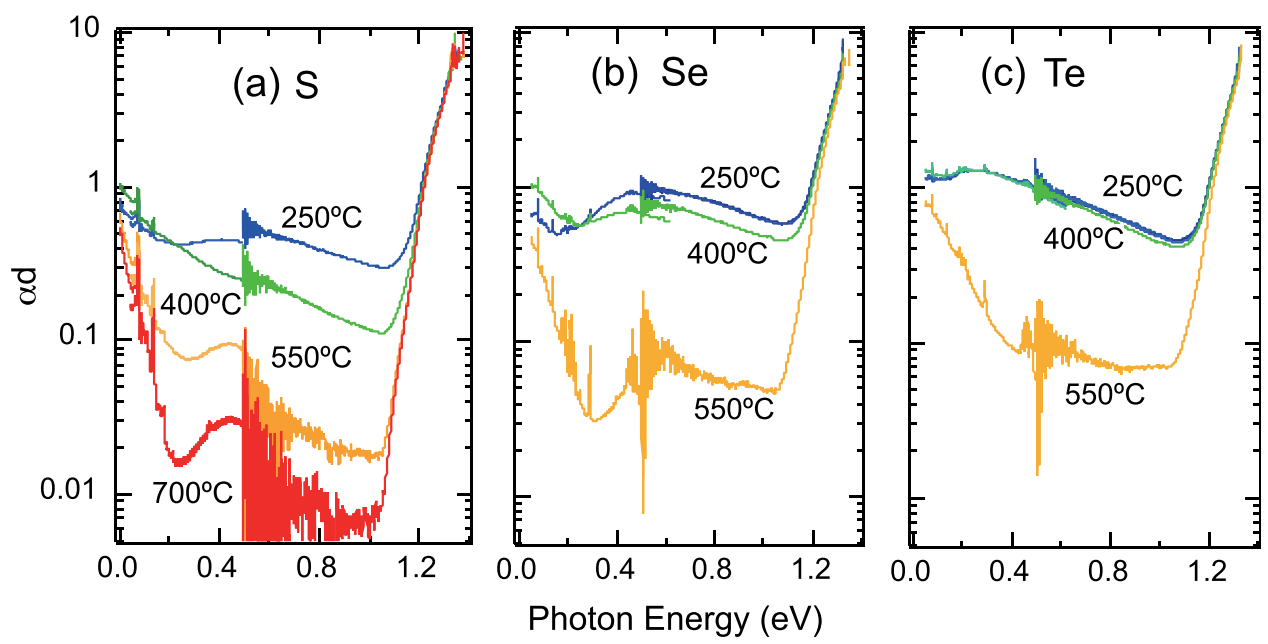

FIG. 2. Effect of thermal annealing on the room temperature optical absorption for (a) sulfur, (b) selenium, and (c) tellurium, all implanted to the same dose of $1 \times 10^{16} \mathrm{~cm}^{-2}$ and laser melted prior to thermal annealing. 

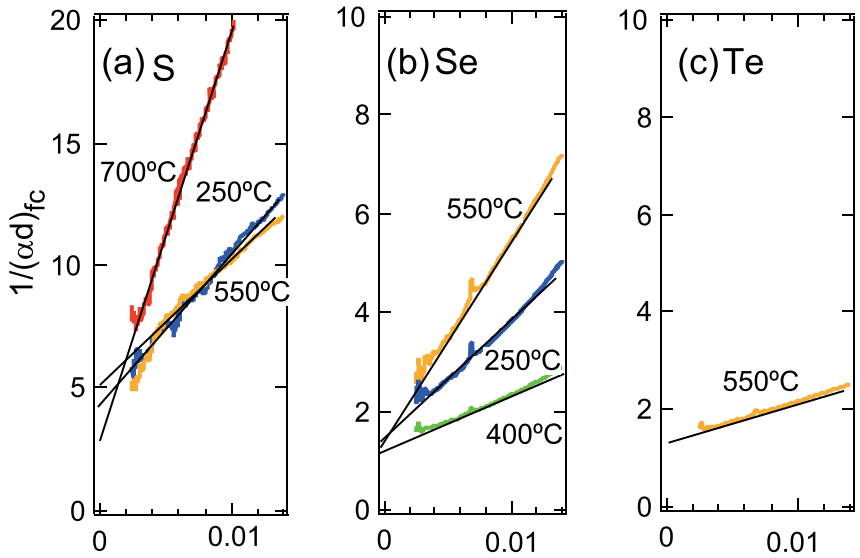

Square of Photon Energy $\left(\mathrm{eV}^{2}\right)$

FIG. 3. Contribution of free carrier absorption in hyperdoped layer at low photon energies and various annealing temperatures for $\mathrm{Si}$ implanted with (a) sulfur, (b) selenium, or (c) tellurium ions to the same dose of $1 \times 10^{16} \mathrm{~cm}^{-2}$, and laser melted. $1 /(\alpha d)_{\mathrm{fc}}$ is plotted against square of photon energy, up to a photon energy of $0.12 \mathrm{eV}$.

According to the Drude model, free carrier absorption is characterized by a quadratic relationship between $1 / \alpha$ and photon energy, $E$

$$
\frac{1}{\alpha}=\frac{\varepsilon_{0} n c}{N e \mu}+\frac{4 \pi^{2} \varepsilon_{0} c n m *^{2} \mu}{N e h^{2}} E^{2},
$$

where $N, e, \mu, m^{*}, \varepsilon_{o}, c$, and $n$ are, respectively, the carrier concentration, electron charge, electron mobility, electron effective mass, permittivity of free space, speed of light, and refractive index. ${ }^{23}$ The reciprocals of $(\alpha d)_{\mathrm{fc}}$ for $1 \times 10^{16} \mathrm{~cm}^{-2}$ hyperdoped samples obtained by the extrapolation and subtraction procedure described above are plotted against the square of the photon energy in Fig. 3 . The results for tellurium samples annealed at 250 and $400{ }^{\circ} \mathrm{C}$ are not shown here because the MIR absorption is large around $0.2 \mathrm{eV}$, as shown in Fig. 2(c), and evaluation of $(\alpha d)_{\mathrm{fc}}$ is not reliable. The plots show straight lines below $0.014 \mathrm{eV}^{2}$ (corresponding to photon energies below $0.12 \mathrm{eV}$ ). The carrier concentration and mobility estimated from these lines using
Eq. (3) are shown in Figs. 4(a) and 4(b). The carrier concentration and mobility for $1 \times 10^{16} \mathrm{~cm}^{-2}$ hyperdoped, unannealed samples are $2.8 \times 10^{19} \mathrm{~cm}^{-3}$ and $100 \mathrm{~cm}^{2} / \mathrm{V} \mathrm{s}$ for sulfur and $3.4 \times 10^{19} \mathrm{~cm}^{-3}$ and $56 \mathrm{~cm}^{2} / \mathrm{V} \mathrm{s}$ for selenium, respectively. These values are consistent with those obtained by Hall effect ${ }^{9,10}$ and (for carrier concentration) $\mathrm{SRP}^{3}$ measurements. Although the pulsed laser melting conditions used to fabricate samples for IR and SRP measurements differ slightly, we have not observed significant differences in electronic properties among the sulfur hyperdoped samples prepared by slightly differing conditions in Refs. 3, 9, and 10. Thus we expect that the differences in electronic properties of the samples prepared for IR and SRP measurement are not significant within the range of variation reported here. In Figs. 4(a) and 4(b), we compare the effect of annealing temperature on carrier concentrations and mobilities of sulfur hyperdoped samples obtained by this method (filled circles) with SRP measurements (crosses). The results obtained by optical and electrical measurements are reasonably consistent, suggesting that the error due to the uncertainty in reflectance is not significant and that linear extrapolation of the tail of the MIR absorption band is reasonable. Certainly, this procedure is reasonable for the samples annealed above $550^{\circ} \mathrm{C}$ because, as shown by the red crosses in Fig. 1(c), the deviation due to reflectance is small, and as shown in Fig. 2, the MIR band absorption is weak.

The contribution of free carrier absorption to the observed spectral shape can be further examined using these results. The free carrier absorption, $(\alpha d)_{\mathrm{fc}}$, of un-annealed samples obtained by this method of linearly extrapolating the tail of the MIR band, is compared in Fig. 5 with the $\alpha d$ spectrum, $(\alpha d)_{\mathrm{fc}}$ SRP, calculated using carrier concentration and mobility obtained by SRP measurement and Eq. (3). For the experimental $\alpha d$ spectra shown in this figure, the contribution of the underlying substrate was subtracted out. Agreement between $(\alpha d)_{\mathrm{fc}}$ and $(\alpha d)_{\mathrm{fc}} \mathrm{SRP}$ is best for the sulfur hyperdoped sample. The differences between measured and calculated values for selenium and tellurium hyperdoped samples, however, do not exceed one order of magnitude. We believe that the results of free carrier absorption obtained by optical and electrical measurements are consistent, given
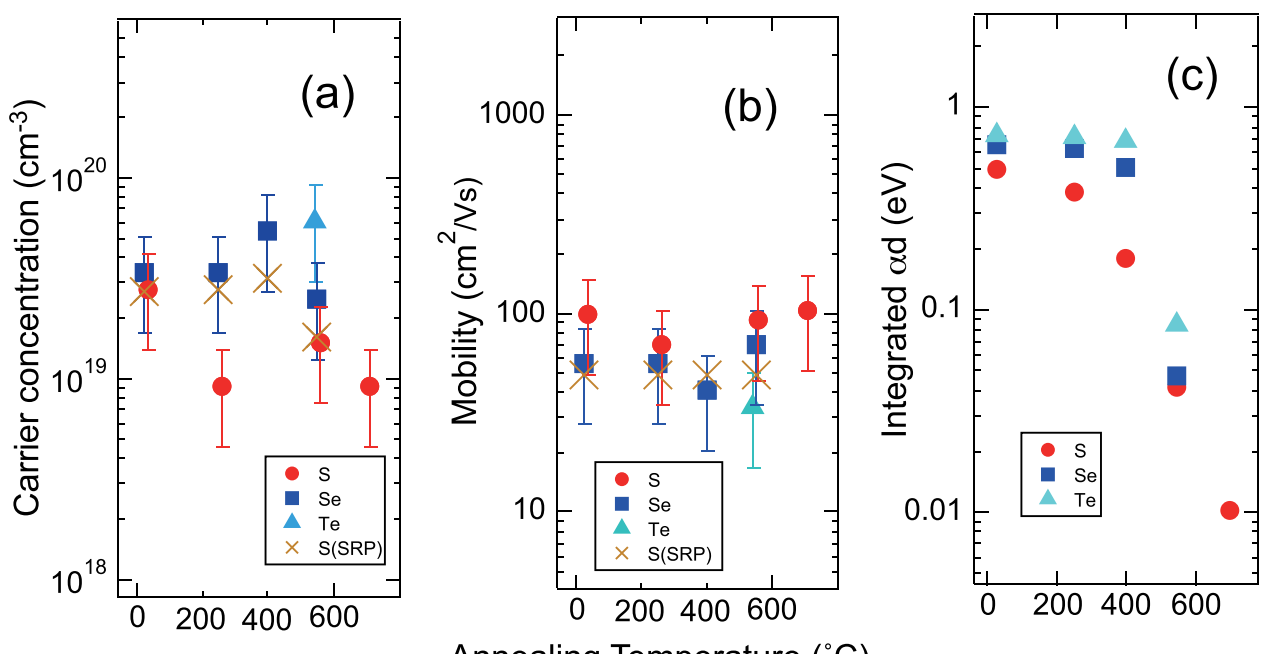

FIG. 4. Annealing temperature dependence of (a) carrier concentration; (b) carrier mobility; (c) integrated intensity of MIR absorption band. Circles, squares, and triangles correspond to the samples implanted to a dose of $1 \times 10^{16} \mathrm{~cm}^{-2}$ with sulfur, selenium or tellurium, respectively. Filled symbols in (a) and (b) represent carrier concentration and mobility estimated from Eq. (3) and Fig. 3 , whereas those obtained by SRP measurements (a) and calculation from the impurity profile per Ref. 21 (b), respectively, are shown as crosses.

Annealing Temperature $\left({ }^{\circ} \mathrm{C}\right)$ 
the procedural uncertainties such as the inferred low- $E$ reflectance and the extrapolation method used to obtain $(\alpha d)_{\text {MIR }}$. Furthermore there is a slight difference in the laser fluence used in samples for optical and SRP measurements as shown in Table II. These results indicate that our interpretation of optical absorption below $0.2 \mathrm{eV}$ as the superposition of MIR absorption and free carrier absorption is reasonable.

As an additional check on our assumptions, we estimate the optical absorption spectra of the MIR band without extrapolation, by subtracting $(\alpha d)_{\mathrm{fc}}^{\mathrm{SRP}}$ from $\alpha d$. The result, which we call $(\alpha d)_{\text {MIR }}{ }^{\text {SRP }}$, is also shown in Fig. 5. Although the results are not perfect, as evidenced by the lack of complete agreement between $(\alpha d)_{\mathrm{fc}}$ and $(\alpha d)_{\mathrm{fc}}{ }_{\mathrm{SRP}}$, it is apparent that the effect of hyperdoping on the MIR absorption band extends down to at least $0.05 \mathrm{eV}$.

Based on the previous SIMS results, ${ }^{3}$ a hyperdoped layer thickness of $250 \mathrm{~nm}$ corresponds to an average sulfur concentration of $3.2 \times 10^{20} \mathrm{~cm}^{-3}$. Using the measured sulfur concentration and carrier concentration and assuming a Boltzmann distribution, the energy level of the donor can be roughly estimated. If all sulfur atoms act individually as single or double donors and carriers are generated by thermal activation of donors, the observed carrier concentration of $\sim 10^{19} \mathrm{~cm}^{-3}$ corresponds to a donor level of about 0.09 (single donor) or 0.1 (double donors) eV below the conduction band, respectively. This agrees well with a calculated binding energy of $117 \mathrm{meV}$ obtained by Winkler from a fit to low temperature Hall effect carrier concentration measurements on lower-dose $\left(10^{14}\right.$ at. $\left./ \mathrm{cm}^{2}\right) \mathrm{S}$ implanted and nanosecond laser-melted silicon-on-insulator samples. ${ }^{24}$ If instead the sulfur atoms compose dimers or trimers the number of donors per sulfur atom is reduced. In such a case, estimation of the number is difficult because the structure of these clusters is unclear. If we assume the number of donors per sulfur

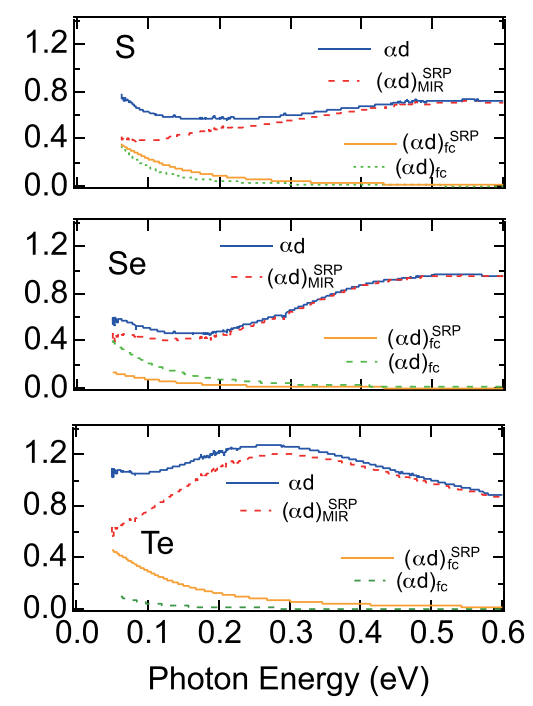

FIG. 5. Effect of free carrier absorption on the observed absorption spectra for un-annealed samples. The result of the measurement $(\alpha d)$, the free carrier absorption obtained by subtracting a linearly extrapolated MIR absorption spectrum $(\alpha d)_{\mathrm{fc}}$, and that calculated by carrier concentration and mobility data obtained by SRP measurement $(\alpha d)_{\mathrm{fc}}$ SRP are plotted as functions of photon energy. The optical absorption spectrum of the MIR band deduced by subtracting $(\alpha d)_{\mathrm{fc}}$ SRP from $\alpha d$ is denoted as $(\alpha d)_{\mathrm{MIR}}$ SRP.
TABLE II. Pulsed laser melting conditions.

\begin{tabular}{|c|c|c|}
\hline \multirow[b]{2}{*}{ Dopant } & \multicolumn{2}{|l|}{ Laser fluence } \\
\hline & IR measurement & SRP measurement \\
\hline $\mathrm{S}$ & $1.7 \mathrm{~J} / \mathrm{cm}^{2}(3$ shots $)+1.8 \mathrm{~J} / \mathrm{cm}^{2}$ (1 shot) & $1.7 \mathrm{~J} / \mathrm{cm}^{2}$ (4 shots) \\
\hline $\mathrm{Se}$ & $1.7 \mathrm{~J} / \mathrm{cm}^{2}$ (3 shots) $+1.8 \mathrm{~J} / \mathrm{cm}^{2}$ (1 shot) & $2.3 \mathrm{~J} / \mathrm{cm}^{2}$ (4 shots) \\
\hline $\mathrm{Te}$ & $1.7 \mathrm{~J} / \mathrm{cm}^{2}$ (3 shots) $+1.8 \mathrm{~J} / \mathrm{cm}^{2}$ (1 shot) & $2.3 \mathrm{~J} / \mathrm{cm}^{2}$ (4 shots) \\
\hline
\end{tabular}

atom decreases to 0.5 by clustering, the depth of donor levels decreases by $0.02-0.03 \mathrm{eV}$ to $0.07 \mathrm{eV}$.

The energy states formed by very low concentrations of sulfur, selenium, or tellurium in Si have been known since the $1980 \mathrm{~s}^{25-27}$ A donor level of about $0.1 \mathrm{eV}$ appears to correspond to that of sulfur-, selenium-, and tellurium-related complexes. However, the origin of the donor level(s) responsible for the observed carrier concentration is not clear due to limited information on the electronic structure of the hyperdoped Si system.

\section{B. Broad MIR absorption band}

A contribution from the thick chalcogen-free substrate is significant above $1.1 \mathrm{eV}$ as shown in Fig. 1(a). The wide range optical absorption spectra of the thin $\mathrm{S}, \mathrm{Se}$, and Tehyperdoped layers, presented in Fig. 6, are determined by subtracting the contribution of the substrate. The strength of the MIR band is related to the chalcogen dose. The optical absorption by localized impurity centers in $\operatorname{solids}^{28,29}$ is described by

$$
N_{e} f=\frac{n m c}{2 \pi^{2} e^{2} \hbar}\left(\frac{E_{0}}{E_{e f f}}\right)^{2} \int \alpha(E) d E
$$

where $N_{e}, m, n, f, \mathrm{c}, E_{0}$, and $E_{\text {eff }}$ are the volumetric density of electrons contributing to impurity absorption, mass of electron, refractive index, oscillator strength, speed of light in vacuum, the average electric field in the dielectric, and the effective field at the center, respectively. The local field correction, $\left(E_{0} / E_{e f f}\right)^{2}$, is approximated as $9 /\left(n^{2}+2\right)^{2}$ for

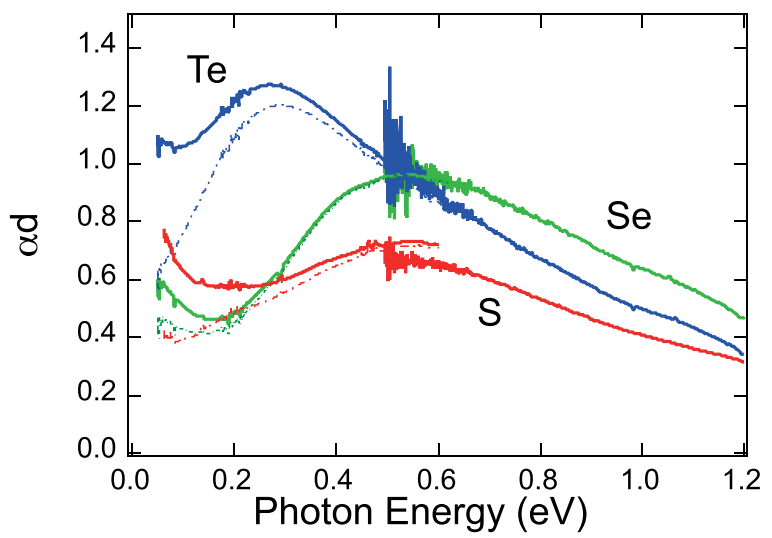

FIG. 6. Optical absorption spectra of $1 \times 10^{16} \mathrm{~cm}^{-2}$ sulfur, selenium, or tellurium implanted and laser hyperdoped layers after subtracting the contribution of the underlying substrate. For comparison, deduced MIR band absorptions, $(\alpha d)_{\text {MIR }}{ }^{\text {SRP }}$, are shown as dotted lines for $\mathrm{S}$ and Te. 
localized centers and unity for sufficiently delocalized centers. Because we measure $\alpha d$ instead of $\alpha$ in this article, we estimate the product $N_{e} f d$ by applying Eq. (4) to the curve for sulfur in Fig. 6. The value of $N_{e} f d$ estimated by this method, using the local field correction to localized centers in Eq. (4), is $9 \times 10^{14} \mathrm{~cm}^{-2}$. If the number of electrons per sulfur atom contributing to this absorption is two and the oscillator strength is unity, $N_{e} f d$ estimated by the retained sulfur dose measured by SIMS should be $2 \times 10^{16} \mathrm{~cm}^{-2}$, which is more than an order of magnitude larger than that obtained by Eq. (4) using the local field correction for localized centers. If, instead, we estimate the value of $N_{e} f d$, using the local field correction for delocalized centers in Eq. (4), we obtain $2 \times 10^{16} \mathrm{~cm}^{-2}$, which is consistent with two electrons per sulfur atom. Furthermore, this result is consistent with the previous reports of delocalization and formation of an impurity band by hyperdoping of chalcogen atoms. ${ }^{10,15} \mathrm{We}$ cannot, however, rule out the possibility that the absorbing centers remain localized, while other effects cause the calculated value of $N_{e} f d$ to be much smaller than the sulfur atom concentration. For example, the limits of integration in Eq. (4) extend, in principle, across the entire absorption band, whereas we integrate only over the region from 0.05 to $1.2 \mathrm{eV}$. The assumption that the average oscillator strength is unity is not valid if significant absorption due to hyperdoping exists above $1.2 \mathrm{eV}$, masked by valence band-to-conduction band absorption.

The attribution of the MIR absorption band to the formation of an impurity band was suggested ${ }^{4}$ when strong absorption was first measured down to $\sim 0.5 \mathrm{eV}$ in these materials. It was also suggested by temperature-dependent Hall effect measurements demonstrating an insulator to metal transition. ${ }^{10,15} \mathrm{Ab}$ initio calculations show the formation of a chalcogen impurity band and its contribution to IR absorption. ${ }^{14,15}$ If the MIR absorption band is indeed due to the formation of an impurity band, broadening of the MIR absorption band with increasing chalcogen concentration is expected. As shown in Fig. 1(a), the MIR band width increases with increasing sulfur dose and thus our experimental results are consistent with the formation of an impurity band. Furthermore, as shown in Fig. 6, the absorption peak energy of the tellurium doped sample is lower than those of sulfur and selenium doped samples, and the absorption intensity of the tellurium doped sample is strongest. These results are qualitatively consistent with the $a b$ initio calculations of the properties of the impurity band. $^{14}$

Note that we cannot rule out the possibility of a different cause, such as interband transitions made allowed by the hyperdoping perturbation of the Si band structure.

\section{Effect of implanted sulfur dose and thermal annealing on carrier concentration and MIR band}

The hyperdoping of sulfur atoms creates both free electrons and MIR-active centers, and they exhibit contrasting behaviors upon thermal annealing. Carrier concentrations obtained by Hall measurements ${ }^{9}$ are plotted in Fig. 1(b) with cross symbols. The preparation method of samples for Hall measurements is the same with that for optical measurements except that they receive only one laser shot-at $1.8 \mathrm{~J} / \mathrm{cm}^{2}$. Fig. 1(c) shows that carrier concentration increases with implantation dose and Fig. 4(a) shows that it depends only weakly on thermal annealing. The SRP data of Fig. 4(a) do show a slight increase in carrier concentration for a $\mathrm{S}$ hyperdoped sample annealed at $400^{\circ} \mathrm{C}$. From Fig. 2(b), $\alpha d$ in the low-energy (free-carrier) region of the spectrum is higher for a Se hyperdoped layer annealed at $400{ }^{\circ} \mathrm{C}$ than for samples annealed at $250{ }^{\circ} \mathrm{C}$ or $550^{\circ} \mathrm{C}$; $\mathrm{S}$ hyperdoped samples show a similar behavior, as shown in Fig. 2(a). These observations suggest that under certain conditions, thermal annealing can increase the carrier concentration and that this may influence the absorption spectrum in the low energy region. Although the carrier concentration is not greatly altered by thermal annealing, the absorption in the MIR band changes substantially as temperature increases. This further confirms that the MIR region of the spectrum is not attributable to free carriers, but to structures that are locally metastable: thermal annealing appears to cause a local structural relaxation in which the MIR centers are deactivated.

\section{CONCLUSIONS}

We measured the near through MIR optical absorption spectra of monocrystalline silicon layers hyperdoped with chalcogen atoms synthesized by ion implantation followed by pulsed laser melting. The observed spectra exhibit both free carrier absorption and a broad MIR absorption band. The center of the MIR absorption band is at a photon energy of about $0.5 \mathrm{eV}$ for $\mathrm{S}$ and Se hyperdoping, and about $0.3 \mathrm{eV}$ for Te hyperdoping. This MIR absorption band is very broad and extends down to at least $0.05 \mathrm{eV}$. The strength of the MIR absorption band is proportional to the implanted sulfur concentration and the width of the MIR absorption band increases with increasing chalcogen concentration. The contribution of free carriers to optical absorption is relatively small but not insignificant below about $0.2 \mathrm{eV}$. The strength of the MIR absorption band drops drastically with increasing annealing temperature over the range $400-800^{\circ} \mathrm{C}$, whereas the carrier concentration and mobility are comparatively insensitive to annealing temperature. The results are consistent with the interpretation that a metastable local atomistic configuration is responsible for the MIR absorption band whereas a more stable, optically inactive configuration is responsible for donating free carriers. These results support the hypothesis that isolated chalcogen impurity levels in the band gap form an impurity band with increasing concentration.

\section{ACKNOWLEDGMENTS}

The authors gratefully acknowledge technical assistance from Yunqing Chen and helpful conversations with D. Recht, M. Winkler, J. Carey, P. Persans, and E. Mazur, and are grateful to D. Recht for assistance with manuscript preparation. M.T. acknowledges the financial support of the Fulbright Program. This research was supported in part by the U.S. Army ARDEC under Contract No. W15QKN-07-P-0092, and 
a Grant-in-Aid for Scientific Research from the Japan Society for the Promotion of Science. The Australian Research Council is also acknowledged for partial financial support.

${ }^{1}$ C. H. Crouch, J. E. Carey, J. M. Warrender, M. J. Aziz, and E. Mazur, Appl. Phys. Lett. 84, 1850 (2004).

${ }^{2}$ C. Wu, C. H. Crouch, L. Zhao, J. E. Carey, R. Younkin, J. A. Levinson, E. Mazur, R. M. Farrell, P. Gothoskar, and A. Karger, Appl. Phys. Lett. 78, 1850 (2001).

${ }^{3}$ B. P. Bob, A. Kohno, S. Charnvanichborikarn, J. M. Warrender, I. Umezu, M. Tabbal, J. S. Williams, and M. J. Aziz, J.Appl. Phys. 107, 123506 (2010).

${ }^{4}$ T. G. Kim, J. M. Warrender, and M. J. Aziz, Appl. Phys. Lett. 88, 241902 (2006).

${ }^{5}$ M. Tabbal, T. Kim, D. N. Woolf, B. Shin, and M. J. Aziz, Appl. Phys. A 98, 589 (2010).

${ }^{6}$ J. E. Carey, C. H. Crouch, M. A. Sheehy, M. Shen, C. M. Friend, and E. Mazur, Opt. Lett. 30, 1773 (2005).

${ }^{7}$ Z. Huang, J. E. Carey, M. Liu, X. Guo, E. Mazur, and J. C. Campbell, Appl. Phys. Lett. 89, 033506 (2006).

${ }^{8}$ A. J. Said, D. Recht, J. T. Sullivan, J. M. Warrender, T. Buonassisi, P. D. Persans, and M. J. Aziz, Appl. Phys. Lett. 99, 073503 (2011).

${ }^{9}$ M. Tabbal, T. G. Kim, J. M. Warrender, M. J. Aziz, B. L. Cardozo, and R. S. Goldman, J. Vac. Sci. Technol. B 25, 1847 (2007).

${ }^{10}$ M. T. Winkler, D. Recht, M.-J. Sher, A. J. Said, E. Mazur, and M. J. Aziz, Phys. Rev. Lett. 106, 178701 (2011).

${ }^{11}$ See www.osti.gov, www.osti.gov/bridge/servlets/purl/984305-ly0Wxh/ for 'Black Silicon Enhanced Thin Film Silicon Photovoltaic Devices', Sionyx, Inc. (2010).
${ }^{12}$ E. Antolín, A. Martí, J. Olea, D. Pastor, G. González-Díaz, I. Mártil, and A. Luque, Appl. Phys. Lett. 94, 042115 (2009).

${ }^{13}$ J. Olea, M. Toledano-Luque, D. Pastor, G. González-Díaz, and I. Mártil, J. Appl. Phys. 104, 016105 (2008).

${ }^{14}$ K. Sánchez, I. Aguilera, P. Palacios, and P. Wahnón, Phys. Rev. B 82, 165201 (2010).

${ }^{15}$ E. Ertekin, M. T. Winkler, D. Recht, A. J. Said, M. J. Aziz, T. Buonassisi, and J. C. Grossman, Phys. Rev. Lett. 108, 026401 (2012).

${ }^{16}$ J. T. Sullivan, R. G. Wilks, M. T. Winkler, L. Weinhardt, D. Recht, A. J. Said, B. K. Newman, Y. Zhang, M. Blum, S. Krause, W. L. Yang, C. Heske, M. J. Aziz, M. Bär, and T. Buonassisi, Appl. Phys. Lett. 99, 142102 (2011).

${ }^{17}$ S. H. Pan, D. Recht, S. Charnvanichborikarn, J. S. Williams, and M. J. Aziz, Appl. Phys. Lett. 98, 121913 (2011).

${ }^{18}$ R. Reitano, P. M. Smith, and M. J. Aziz, J. Appl. Phys. 76, 1518 (1994).

${ }^{19}$ E. D. Palik, Handbook of Optical Constants of Solids (Academic Press, Inc., New York, 1985).

${ }^{20}$ J. I. Pankove, Optical Processes in Semiconductors (Dover, New York, 1971).

${ }^{21}$ G. Kaiblinger-Grujin, T. Grasser, and S. Selberherr, Simulation of Semiconductor Processes and Devices (Springer, Leuven, 1998).

${ }^{22}$ B. R. Tull, M. T. Winkler, and E. Mazur, Appl. Phys. A 96, 327 (2009).

${ }^{23}$ Y. Mishima, M. Hirose, and Y. Osaka, J. Appl. Phys. 51, 1157 (1980).

${ }^{24}$ M. T. Winkler, Ph.D. dissertation, Harvard University, 2009.

${ }^{25}$ E. Janzén, R. Stedman, G. Grossmann, and H. G. Grimmeiss, Phys. Rev. B 29, 1907 (1984)

${ }^{26}$ J. C. Swartz, D. H. Lemmon, and R. N. Thomas, Solid State Commun. 36, 331 (1980).

${ }^{27}$ P. Wagner, C. Holm, E. Sirtl, R. Oeder, and W. Zulehner, Festkorperprobleme XXIV, 191 (1984)

${ }^{28}$ A. Smakula, Z. Phys. 59, 603 (1930).

${ }^{29}$ L. Vina and M. Cardona, Phys. Rev. B 29, 6739 (1984). 